\title{
Measurement of parasitological data by quantitative real-time PCR from controlled human malaria infection trials at the Walter Reed Army Institute of Research
}

\author{
Edwin Kamau*, Saba Alemayehu, Karla C Feghali, Jack Komisar, Jason Regules, Jessica Cowden \\ and Christian F Ockenhouse
}

\begin{abstract}
Background: The use of quantitative real-time PCR ( $(\mathrm{PCR}$ ) has allowed for precise quantification of parasites in the prepatent period and greatly improved the reproducibility and statistical power of controlled human malaria infection (CHMI) trials. Parasitological data presented here are from non-immunized, control-challenged subjects who participated in two CHMI trials conducted th the Walter Reed Army Institute of Research (WRAIR).

Methods: Standardized sporozoite challenge was achieved through the bite of five Anopheles stephensi mosquitoes infected with the 3D7clone of the NF54 strain of Plasmodium falciparum. Blood smears were scored positive when two unambiguous parasites were found. Analysis of parasitological PCR data was performed on log-transformed data using an independent sample t-test when comparing the two studies. The multiplication rate of blood-stage parasites was estimated using the linear model.

Results: On average, parasites were detected 4.91 days ( $95 \% \mathrm{Cl}=4.190$ to 5.627 ) before smears. The earliest parasites were detected within 120 hours (5.01 days) after challenge. Parasite densities showed consistent cyclic patterns of blood-stage parasite growth in all volunteers. The parasite multiplication rates for both studies was 8.18 ( $95 \% \mathrm{Cl}=6.162$ to 10.20). Data showed that at low parasite densities, a combination of sequestration and stochastic effects of low copy number DNA may impact qPCR detection and the parasite detection limit.

Conclusion: Smear positive is an endpoint which antimalarial rescue is imperative whereas early detection of parasitological data by qPCR can allow for better anticipation of the endpoint. This would allow for early treatment to reduce clinical illness and risk for study participants. To use qPCR as the primary endpoint in CHMI trials, an algorithm of two positives by $\mathrm{QPCR}$ where one of the positives must have parasite density of at least 2 parasites $/ \mu \mathrm{L}$ is proposed.
\end{abstract}

\section{Background}

Controlled human malaria infection (CHMI) is increasingly being used to assess the efficacy of malaria vaccines as well as to evaluate antimalarial drug candidates [1,2]. Data obtained from CHMI are critical in the decisionmaking process of whether or not to proceed with more costly Phase IIb field trials [1]. CHMI trials allow for detailed evaluation of parasite growth kinetics and provide

\footnotetext{
*Correspondence: edwin.kamau@us.army.mil

Military Malaria Research Program, Malaria Vaccine Branch, Walter Reed Army Institute of Research, 503 Robert Grant Ave, Silver Spring, Maryland, USA
}

an opportunity to characterize immunological responses $[1,2]$, which can be informative for further optimization of a vaccine or drug candidate. Studies have shown a high correlation between natural and experimental infections, which further validates the importance of using CHMI in testing new vaccines or drugs [1].

In CHMI trials, subjects are inoculated (challenged) with either Plasmodium falciparum or Plasmodium vivax sporozoites from bites of infectious, laboratory-reared, female anopheline mosquitoes. After being challenged, subjects are closely monitored for signs and symptoms of malaria 
such as headache, myalgia and fever. Screening for bloodstage parasites is performed by the examination of blood smears at regular intervals starting five to seven days post challenge [3]. Subjects are treated with antimalarial drugs when patent parasitaemia is confirmed by blood smears following criterion set forth in the study protocol. The detection threshold of parasites on Giemsa-stained thick films is about two to 20 parasites $/ \mu \mathrm{L}$ depending on the expertise of the microscopist and the number of high-powered fields examined on a thick blood film. The comparative prepatent period of treated subjects and control subjects is used to assess efficacy of the vaccine or drug being tested [3].

The development of quantitative real-time PCR (qPCR) and other molecular techniques, which are more sensitive than microscopy has allowed for precise quantification of parasites during the prepatent period [4-6]. The qPCR data can be used to estimate liver parasite load (for preerythrocytic vaccines) or blood-stage multiplication rate (for erythrocytic vaccines), providing additional detailed information on the efficacy of the vaccine or drug candidate [7-10]. The mean prepatent period for control subjects challenged with sporozoites by mosquito bite is 11 days (ranges seven to 20 days), with almost $100 \%$ of subjects bitten by five infectious mosquitoes developing patent parasitaemia [11-13]. Once the parasite emerges from the liver, the number in peripheral blood depends on multiplication rates and sequestration of parasites. Previous studies have detected parasites by PCR as early as 5.5 days after sporozoite challenge [6], with parasites detected by qPCR on average two to four days before detection by blood smears $[3,6]$.

The use of molecular analysis has enhanced the reproducibility and statistical power of CHMI [1]. Harmonization of CHMI study methodology will further strengthen the use of molecular analysis and will allow more accurate comparison of studies conducted in different centres [3]. Further, a recent study showed that small CHMI trials even with small number of subjects are sufficiently powered to detect protective biological effects induced by pre-erythrocytic and/or blood-stage candidate vaccines if parasitaemia is measured daily by qPCR [14]. This study set-out to perform descriptive analysis of parasitological dataset from two CHMI trials recently conducted at the Walter Reed Army Institute of Research (WRAIR) in Silver Spring, MD, USA. The data presented here is from non-immunized, controlchallenged volunteers only.

\section{Methods}

\section{Study subjects}

Samples used for analysis in this study were from infectivity control challenge subjects enrolled in two different studies conducted at WRAIR in 2012. All the subjects developed infection following challenge. These subjects did not receive any investigational product or licensed antimalarial medication prior to challenge by the bite of infectious mosquitoes. Participants were malaria-naïve adult males and females from 18-50 years of age. Protocols for both studies were approved by the WRAIR Institutional Review Board and by the Human Subjects Research and Review Board of the Surgeon General of the US Army at Fort Detrick, Maryland. Participants in both studies provided written, informed consent before screening and enrolment and had to pass an assessment of understanding.

\section{Infection procedures}

Standardized sporozoite challenge was achieved through the bite of five Anopheles stephensi mosquitoes infected with the 3D7clone of the NF54 strain of P. falciparum. In both studies, 3D7 was prepared from the same master seed. Parasitaemia was detected by the routine daily examination of blood smears from day 5 to day 18 following challenge. Blood smears were analysed every two days from day 20 until day 28 post-challenge for any individual who had not yet become parasitaemic. Food and Drug Administration (FDA) approved oral antimalarials licensed in the USA were administered under directly observed therapy to treat uncomplicated malaria as soon as parasitaemia was detected by microscopy.

\section{Sample collection and processing}

Samples were routinely collected every morning for blood smears and qPCR. In addition, samples were collected if a subject became symptomatic outside of scheduled, routine collection times. However, samples collected outside the routine times were not analysed in this study. Samples were collected in EDTA blood tubes and aliquoted for immediate use, or immediately stored in $-20^{\circ} \mathrm{C}$. For microscopic analysis, thick smears were prepared by spreading $10 \mu \mathrm{L}$ of blood on a slide. The $10 \mu \mathrm{L}$ of blood was smeared to make a $1 \mathrm{~cm} \times 2 \mathrm{~cm}$ rectangle, with two such rectangles per slide. To read the smear, five passes across the $1-\mathrm{cm}$ dimension of the smear were made for asymptomatic individuals and 21 passes were made for symptomatic individuals, unless the individual was found to be positive before the completion of the examination. The volume of blood examined by making five passes was $0.55 \mu \mathrm{L}$. Slides were examined by two independent readers under oil immersion at $1000 \times$ magnification. Blood smears were scored positive when two unambiguous parasites were found. The same team of microscopists was responsible for smear analysis in both studies.

\section{Real-time PCR assay}

The qPCR analysis was done in real-time using reagents, procedures and methods as previously described $[15,16]$. 
The assay characteristics, including the limit of detection, have been described previously [17]. Samples were analysed using the genus-specific PLU assay and endogenous control RNaseP assay, which was performed as a multiplex assay. The WHO International Standard for P. falciparum DNA (obtained from National Institute for Biological Standards and Control; NIBSC, Hertfordshire, UK. Referred to herein as NIBSC standard), plasmid DNA or combination of both was used as standard for quantification of the parasite density in all the qPCR assays performed. Details of the reagents and analysis methods have been described previously $[15,16]$. Briefly, DNA was purified from $200 \mu \mathrm{L}$ whole blood and eluted in $200 \mu \mathrm{L}$ elution buffer or water using EZ1 automated purification system (Qiagen, Valencia, CA, USA) with the EZ1 DNA blood kit (Qiagen) following the manufacturer's recommendation. For qPCR assay, $1 \mu \mathrm{L}$ DNA was added in $4 \mu \mathrm{L}$ master mix. Each assay was run on a 96-well plate with NIBSC standard. The performance of the NIBSC standard and RNaseP for individual assays was assessed to determine the success of the analysis. The amplification plot for each assay was individually assessed to ensure true amplification of the assay when $\mathrm{C}_{\mathrm{q}}$ (quantification cycle, can also be referred to as the threshold cycle $\left[C_{t}\right]$ ) values were obtained. The qPCR assays were performed within four hours of blood collection.

\section{Data analysis}

Statistical analyses were performed using Graph-Pad Prism (San Diego, CA, USA) and STATA software version 10 (College Station, TX, USA). Prepatent period was defined as length of time between challenge and detection of a positive blood smear. Analysis of parasitological PCR data was performed on log-transformed data using an independent sample t-test when comparing the two studies. The multiplication rate of blood-stage parasites was estimated using the linear model as recently described [18]. The cycles of parasite growth were estimated as follows: the first cycle started the first day parasites were detected until day 8 , the second cycle was day 9-10, the third cycle was day 11-12, and the fourth cycle was day 13-14 where applicable.

\section{Results}

\section{Time to parasitaemia}

Data from 16 unvaccinated infectivity control subjects enrolled in two different malaria vaccine trials at the WRAIR were analysed by qPCR. Table 1 shows the mean number of days before detection of parasites in both studies by blood smears and qPCR. The earliest parasites detected were in a subject from the second study where parasites were detected by qPCR 120 hours (5.01 days) after the time of challenge, with a parasite density of 0.174 parasite $/ \mu \mathrm{L}$. Parasites were detected by qPCR in all study subjects within seven days following challenge. In the first study, parasites were detected on average 4.34 days ( $95 \% \mathrm{CI}=3.70$ to 4.98 ) before smears, whereas in the second study, parasites were detected on average 6.17 (95\% CI $=4.57$ to 7.77 ) days before smears. Figure $1 \mathrm{~A}$ and $\mathrm{B}$ are survival curves showing the per cent of subjects negative by both smears and qPCR for first and second study.

\section{Parasite density}

Parasite densities showed consistent cyclic patterns of blood-stage parasite growth in all volunteers from both studies. Figure 2 shows the geometric mean of parasite density from both studies. Parasites in the second study were detected sooner by qPCR compared to the first study and at a lower density. Parasite geometric mean density first cycle was 0.444 parasites $/ \mu \mathrm{L}(95 \% \mathrm{CI}=0.274$ to 0.718$)$ for the first study and 0.202 parasites/ $\mu \mathrm{L}(95 \%$ $\mathrm{CI}=0.107$ to 0.383 ) for the second study. Geometric mean parasite density by smears for the first study was 3.29 parasites/ $\mu \mathrm{L}$ (95\% CI 2.00 to 5.42$)$ and $1.68(95 \%$ $\mathrm{CI}=0.97$ to 2.92 ) for the second study. Geometric mean parasite density by qPCR analysis on the first day of qPCR positivity for the first study was 0.394 parasites $/ \mu \mathrm{L}$ (95\% $\mathrm{CI}=0.272$ to 0.572$)$ and 0.174 parasites $/ \mu \mathrm{L}(95 \%$ $\mathrm{CI}=0.079$ to 0.383 ) for second study. Interestingly, the geometric mean parasite density by qPCR at the day of smear positive for first study was 23.92 parasites $/ \mu \mathrm{L}$ (95\% CI $=16.43$ to 34.80$)$ and 35.74 parasites/ $\mu \mathrm{L}(95 \%$ $\mathrm{CI}=25.43$ to 50.23 ) for second study.

Table 1 Time to parasitaemia (days), showing the number of subjects from the first and second study

\begin{tabular}{lllllllll}
\hline & Mean & Std Dev & Max & Min & Median & 25\% & 75\% & P Value \\
\hline First study $(\mathbf{n}=\mathbf{1 1})$ & & & & & & & & \\
Smear & 11.34 & 0.93 & 13.02 & 10.11 & 10.96 & 10.91 & 11.97 & 0.0001 \\
qPCR & 7.01 & 0.07 & 7.12 & 6.91 & 6.98 & 6.95 & 7.07 \\
Second study $(\mathbf{n = 5 )}$ & & & & & & & \\
Smear & 12.54 & 1.33 & 14.00 & 10.96 & 11.98 & 11.43 & 13.94 \\
qPCR & 6.38 & 0.89 & 7.01 & 5.01 & 6.93 & 5.47 & 7.00 & 0.0004 \\
\hline
\end{tabular}




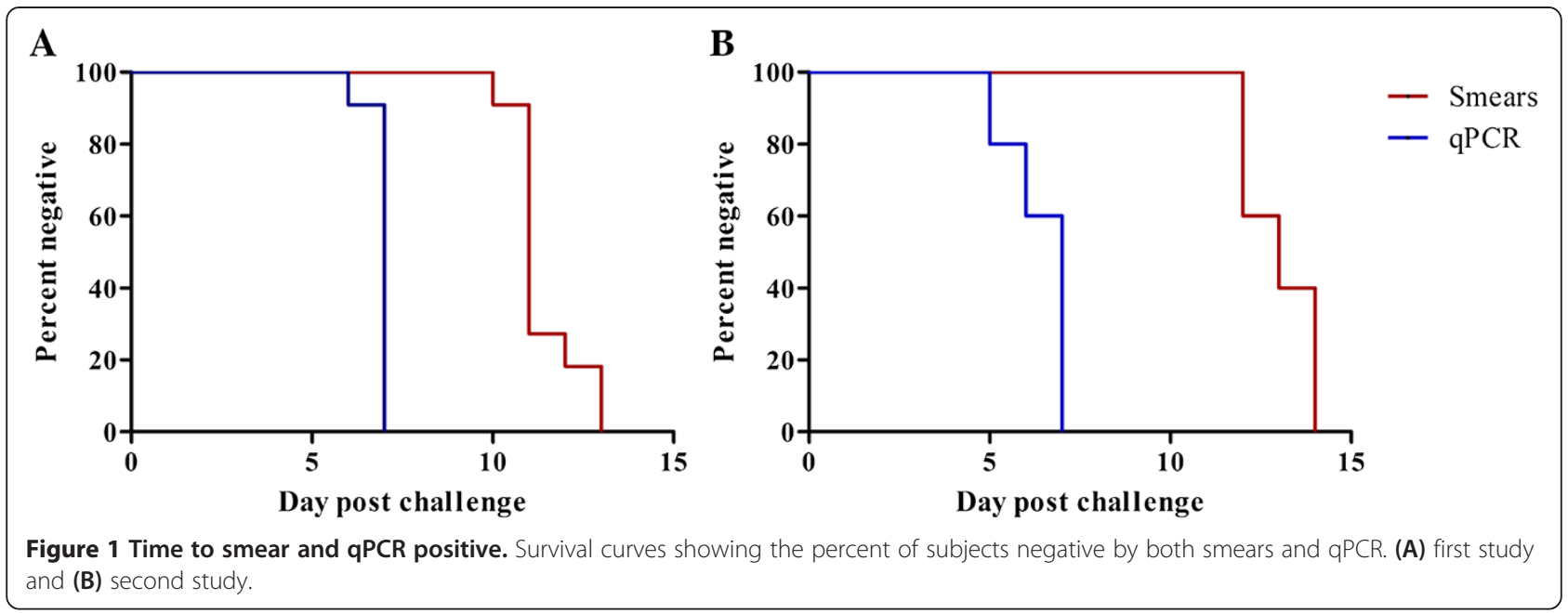

\section{Parasite multiplication rate and growth}

Figure 3 shows parasite multiplication rate (PMR) for the first and second study. The mean PMR for first and second studies were 8.26 (range $=3.5-13.37$ ) and 8.00 (range $=3.98-13.77$ ), respectively. However, parasites in the second study, which were detected sooner and at a lower parasite density, remained low until day 11 (Figure 2). The geometric mean parasite density on day 11 was 22.57 parasites $/ \mu \mathrm{L}(\mathrm{CI}=15.37-33.15)$ for the first study compared to 2.02 parasites $/ \mu \mathrm{L}(\mathrm{CI}=0.37-11.03)$ for second study. This is reflected by the fact that on day 10 and 11, eight out of 11 (seven on day 11) subjects were found parasite positive by smear for the first study whereas for the second study, subjects did not become smear positive until day 12 (two subjects), day 13 (one subject) and day 14 (two subjects). Table 2 shows the geometric mean parasite density in parasites $/ \mu \mathrm{L}$ in the first, second, third, and fourth cycles for first and second study. Figure 4A and $\mathrm{B}$ show the geometric mean parasite density in parasites $/ \mathrm{mL}$ per multiplication cycle for individual subjects. Data clearly indicate that growth rates for the second study were initially slow in the first and second or third cycle but increased rapidly in the third or fourth cycle.

\section{Effect of parasite sequestration in qPCR detection}

The numbers of parasites in peripheral blood depend on multiplication rates and sequestration. At low parasite density, stochastic or random sampling effects of low copy number of DNA during PCR amplification leads to fluctuation of results between replicate analyses. A combination of sequestration and stochastic effects may impact qPCR detection and the $\mathrm{C}_{\mathrm{q}}$ values obtained at low densities. Table 3 shows $\mathrm{C}_{\mathrm{q}}$ values from two subjects for whom qPCR analysis was repeated in eight replicates for two consecutive days. The initial qPCR runs were done in triplicate (shown in bold). The qPCR assay for Subject ID 01 performed on 02FEB, demonstrates stochastic effects due to low parasite density. When the qPCR assay was initially run in triplicate on 02FEB, only one $\mathrm{C}_{\mathrm{q}}$ value out of the three replicates was detected at cycle 42.08 , which is outside the set assay limit of $\mathrm{C}_{\mathrm{q}}$ value of

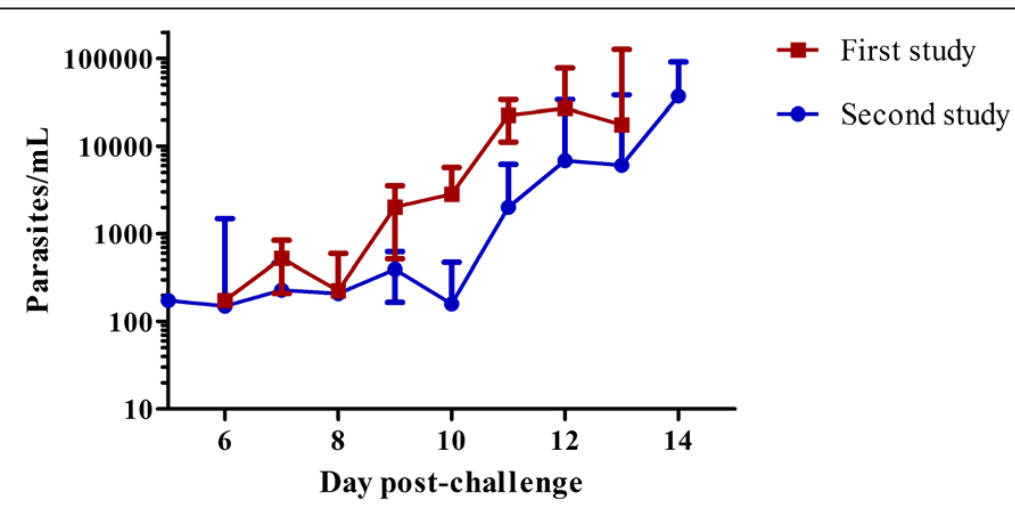

Figure 2 Parasite density as measured by qPCR. The geometric mean is shown from both studies with 95\% confidence intervals, from the day which qPCR was initiated (day 5 after challenge) until the day the subjects became smear positive and treatment was initiated. 


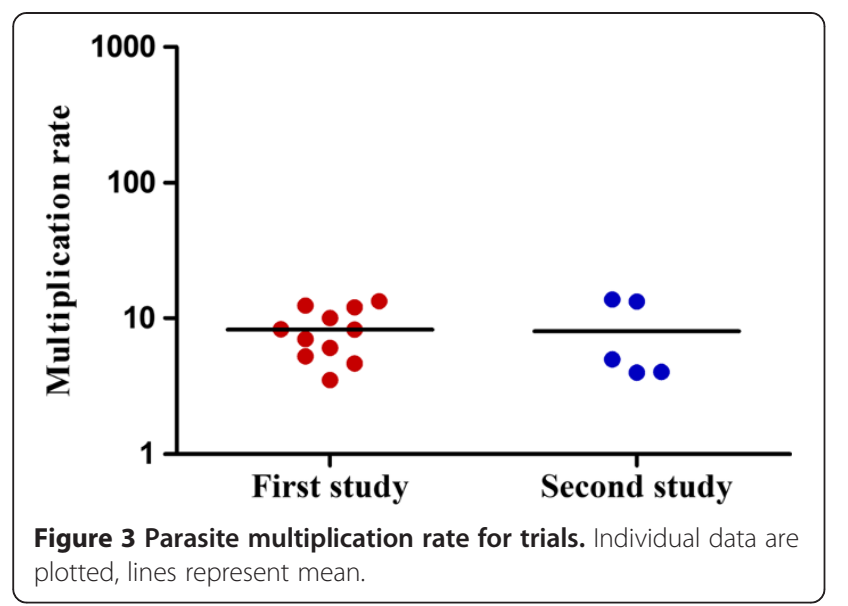

40 , and therefore it was considered there was no amplification. However, when repeated in eight replicates, five $\mathrm{C}_{\mathrm{q}}$ values were recorded with standard deviation (SD) of 0.983 . As the parasite multiplied and density increased (24 hours), all $\mathrm{C}_{\mathrm{q}}$ values were within the detectable range (with lower $\mathrm{SD}$ ) the following day. When initially run in triplicate, subject ID 02 had one $\mathrm{C}_{\mathrm{q}}$ value out of the three replicates detected on 02FEB at cycle 34.47 but none was detected on O3FEB. However, when repeated in eight replicates, five $C_{q}$ values were recorded on 02FEB and four $\mathrm{C}_{\mathrm{q}}$ values were recorded on 03FEB. These data clearly demonstrate that a negative $C_{q}$ value can be due to a lack of parasites in the sample, low parasite density because it is early in the parasite life cycle, low numbers of parasites due to sequestration, or the stochastic effects of low copy number DNA template, which demonstrates the limits of qPCR (molecular) assays.

\section{Discussion}

This study show detailed estimations of critical parameters in the parasite life cycle from CHMI studies conducted at WRAIR using qPCR measurements of parasitaemia in infectivity control (non-vaccinated), malaria-naive (non-immune) volunteers. Parasite analysis was done in real time and results were available within four to six hours from the time of blood collection. Parasites were detected in all volunteers who eventually developed malaria within seven days of the challenge, with the earliest detection being at 5.01 days. Previous studies have demonstrated parasites are detected by $\mathrm{qPCR}$ on average of two to four days before microscopy $[3,6]$. Interestingly, parasites were detected on average 6.17 days before microscopy in the second study. In one of the subjects, parasites were detected 7.93 days before detection by microscopy. This is the largest interval that has been reported in CHMI between qPCR positivity and blood smear parasite detection. In the first study, parasites were detected on average 4.34 days before microscopy. In both studies, there were no false qPCR positives based on data obtained from infectivity controls and vaccine recipients (data to be reported elsewhere).

In CHMI studies, the primary endpoint of blood-stage infection is the first detection of parasites by microscopy. Since CHMI studies mostly recruit malaria-naïve, healthy volunteers, there has been increasing discussion about whether qPCR should replace microscopy as the primary endpoint for clinical diagnosis in order to reduce malaria symptomatology, clinical illness and risk for study participants. Use of qPCR for endpoint diagnosis with the first appearance of PCR positive sample may be suitable for pre-erythrocytic (liver stage) vaccines. However, it would be less useful in the evaluation of vaccines and drugs with blood-stage activity as efficacy for these can only be obtained by evaluating blood-stage parasite growth over a sufficient period of time [14]. Treatment based on qPCR would decrease the time window required for detailed analysis and estimation of critical parameters in the parasite life cycle required for measuring the efficacy of blood-stage vaccines [3]. Development of a qPCR treatment algorithm is therefore critical in keeping the time window long enough to evaluate vaccine efficacy yet short enough to treat volunteers in time (before they would be smear positive) to reduce clinical illness. In this study, the geometric mean parasite density by qPCR on the day of first positive blood smear was 23.92 parasites $/ \mu \mathrm{L}(95 \% \mathrm{CI}=16.43$ $34.80)$ and 35.74 parasites $/ \mu \mathrm{L}(95 \% \mathrm{CI}=25.43-50.23)$ for the first and second study, respectively. The geometric mean parasite density by qPCR on the day prior to the first positive smear was 5.78 parasites $/ \mu \mathrm{L}(95 \% \mathrm{CI}=$ $2.92-11.43)$ and 6.12 parasites $/ \mu \mathrm{L}(95 \% \mathrm{CI}=1.87-20.00)$ for first and second study, respectively. In a study by Roestenberg et al. which compared parasitological data from CHMI studies, the lowest geometric mean peak parasitaemia detected was 7.08 parasites/ $\mu \mathrm{L}$ [10]. In another study, using pooled data, the geometric mean parasitaemia and second and third cycles were determined to be 4.49 and 11.37 parasites $/ \mu \mathrm{L}$, respectively [14]. Most molecular assays used in CHMI studies have a threshold of about 0.02 parasites/ $\mu \mathrm{L}[4,6,17,19]$. To

Table 2 Parasite cycles (parasite/ $\mu \mathrm{L}$ )

\begin{tabular}{lllll}
\hline & 1st cycle $(\mathbf{9 5} \% \mathrm{Cl})$ & 2nd cycle $\mathbf{( 9 5 \% ~ C l )}$ & 3rd cycle (95\% Cl) & 4th cycle $\mathbf{( 9 5 \% ~ C l )}$ \\
\hline First study & $0.44(0.27$ to 0.72$)$ & $2.41(1.33$ to 4.37$)$ & $24.55(16.87$ to 35.73$)$ & Nil \\
Second study & $0.20(0.11$ to 0.38$)$ & $0.29(0.14$ to 0.64$)$ & $3.71(0.41$ to 33.86) & 14.15 (3.36 to 59.66) \\
\hline
\end{tabular}




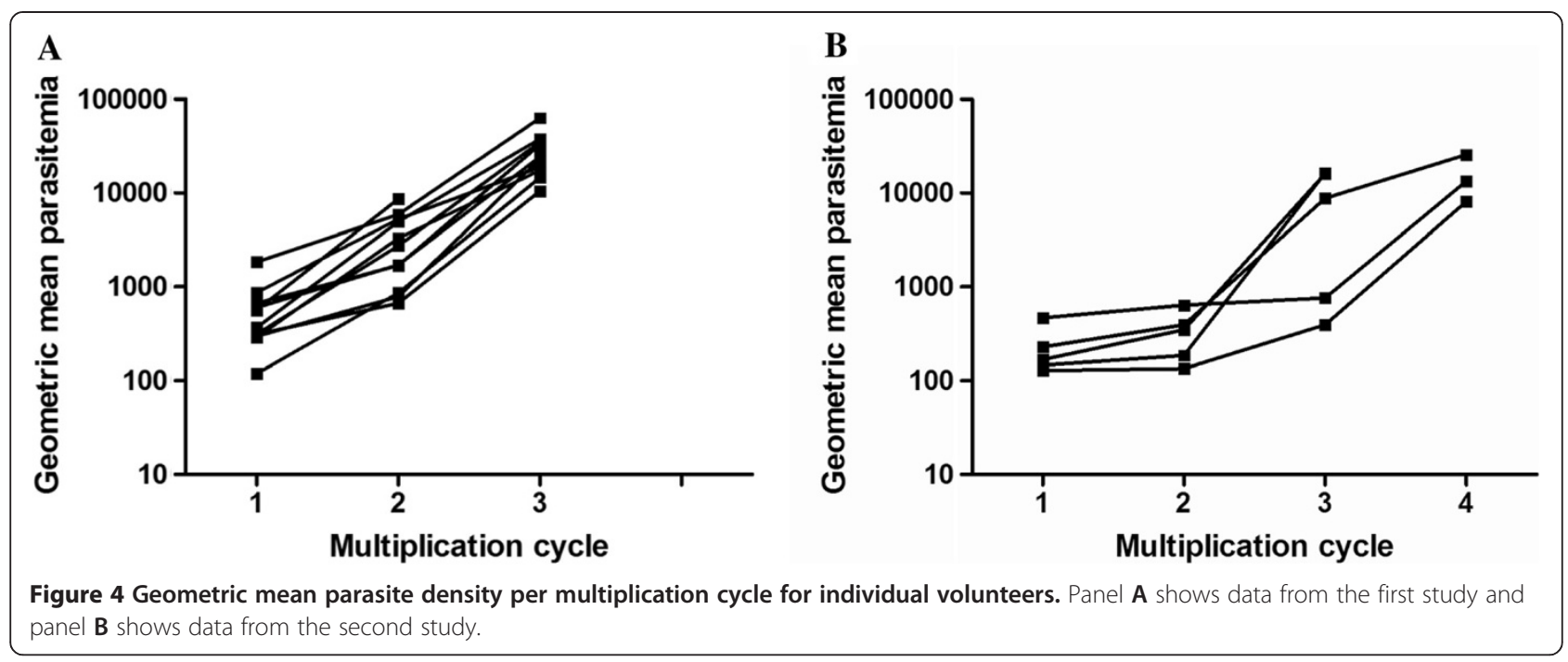

Table 3 Effect of parasite sequestration in QPCR detection

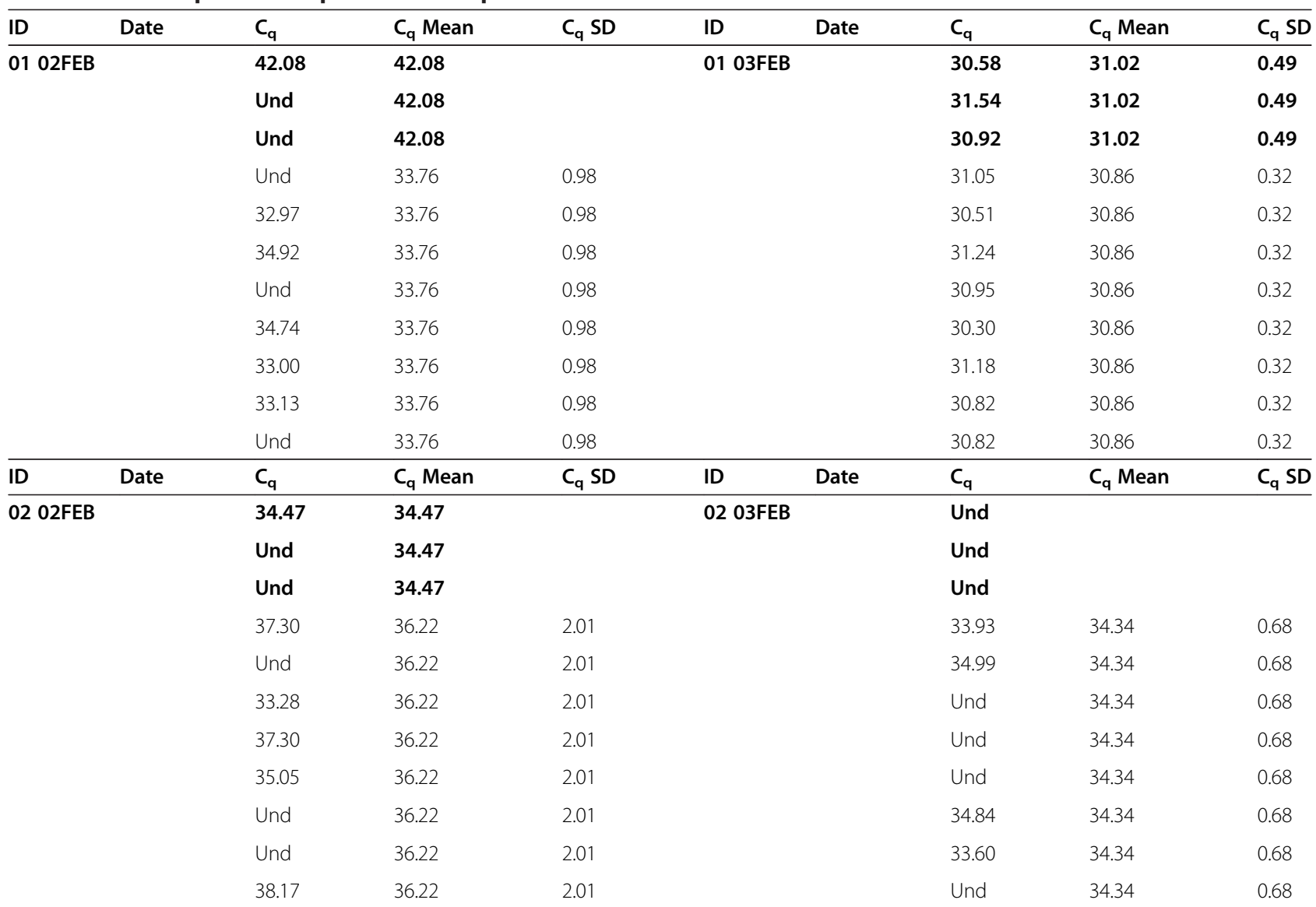


use qPCR for end-point diagnosis in CHMI, an algorithm of two positives, not necessarily on consecutive days (because of parasite sequestration), where one of the positives must have parasite density of at minimum two parasites $/ \mu \mathrm{L}$ is proposed. This is a parasite density that is well within range as detected by qPCR at the day of positive smears in this and other studies. This will allow enough time window for analysis of the effect of the vaccine while still allowing initiation of antimalarial treatment one to two days before becoming smear positive. For this algorithm to be effective however, it is important that CHMI centres use harmonized qPCR analysis and common reference standard DNA for determination of parasite density. In addition, as more data are collected from more centres, the recommendations regarding minimum parasite density should be adjusted.

Inter-individual variation is probably the most important factor in determining the sensitivity of sporozoite challenge trials, which might be influenced by the parasite inoculum size, parasite fitness, and human (innate) immune factors [14]. Use of malaria-naïve volunteers in CHMI studies helps reduce variance due to immune factors. However, parasite inoculum size and parasite fitness is still an issue in sporozoite challenge trials. The variability in prepatent period, parasite growth rates and densities found between first and second study could be due to the infectivity of the sporozoites, the number of sporozoites per mosquito, or the number of sporozoites inoculated by the bites of five infected mosquitoes. This has been shown to vary from centre to centre, between trials at the same centre, and even between individuals in the same trial at the same centre [20]. Although it has been suggested that the use of vialed sporozoites administered by needle and syringe for challenge could reduce these variations $[3,20]$, it is important to be cautious since this is not a natural route of sporozoite infection and will undoubtedly have immune response implications [1]. In addition, it will not address the issues of variation in parasite fitness and innate host variability.

\section{Conclusion}

This study demonstrates reproducibility of CHMI studies conducted at the WRAIR and the use of qPCR for measurements of parasitaemia. An algorithm that can be used for $\mathrm{qPCR}$ as an endpoint diagnosis has been proposed. Use of $\mathrm{qPCR}$ as an endpoint diagnosis is likely to appeal to FDA and other authorities because it will reduce malaria symptomatology, clinical illness and risk for study participants. However, all the benefits of using qPCR as an endpoint diagnosis must be weighed against the legacy of using smears as the endpoint and the backward compatibility with data collected from previous studies.
Competing interests

The authors declare that they have no competing interests.

\section{Authors' contributions}

EK and CFO conceived the project idea. EK, SA KCF designed and executed the experiments. JK was responsible for microscopy work. JR and JC were the principal investigators of the clinical trials. EK wrote the manuscript. SA, $\mathrm{KCF}, J \mathrm{~K}, \mathrm{JR}$ and JC reviewed the manuscript. All authors approved the final version of the manuscript.

\section{Acknowledgments}

We would like to thank Dr Philip Bejon of Wellcome Trust, Kilifi Kenya for his critical review of the manuscript. Material has been reviewed by the Walter Reed Army Institute of Research. There is no objection to its presentation and/or publication. The opinions or assertions contained herein are the private views of the authors, and are not to be construed as official, or as reflecting true views of the Department of the Army or the Department of Defense.

Received: 2 April 2014 Accepted: 14 July 2014

Published: 28 July 2014

\section{References}

1. Sauerwein RW, Roestenberg M, Moorthy VS: Experimental human challenge infections can accelerate clinical malaria vaccine development. Nat Rev Immunol 2011, 11:57-64.

2. McCall MB, Netea MG, Hermsen CC, Jansen T, Jacobs L, Golenbock D, van der Ven AJ, Sauerwein RW: Plasmodium falciparum infection causes proinflammatory priming of human TLR responses. J Immuno/ 2007, 179:162-171.

3. Moorthy VS, Diggs C, Ferro S, Good MF, Herrera S, Hill AV, Imoukhuede EB, Kumar S, Loucq C, Marsh K, Ockenhouse CF, Richie TL, Sauerwein RW: Report of a consultation on the optimization of clinical challenge trials for evaluation of candidate blood stage malaria vaccines, 18-19 March 2009, Bethesda, MD, USA. Vaccine 2009, 27:5719-5725.

4. Andrews L, Andersen RF, Webster D, Dunachie S, Walther RM, Bejon P, Hunt-Cooke A, Bergson G, Sanderson F, Hill AV, Gilbert SC: Quantitative real-time polymerase chain reaction for malaria diagnosis and its use in malaria vaccine clinical trials. Am J Trop Med Hyg 2005, 73:191-198.

5. Felger I, Genton B, Smith T, Tanner M, Beck HP: Molecular monitoring in malaria vaccine trials. Trends Parasitol 2003, 19:60-63.

6. Hermsen CC, Telgt DS, Linders EH, van de Locht LA, Eling WM, Mensink EJ, Sauerwein RW: Detection of Plasmodium falciparum malaria parasites in vivo by real-time quantitative PCR. Mol Biochem Parasitol 2001, 118:247-251.

7. Bejon P, Andrews L, Andersen RF, Dunachie S, Webster D, Walther M, Gilbert SC, Peto T, Hill AV: Calculation of liver-to-blood inocula, parasite growth rates, and preerythrocytic vaccine efficacy, from serial quantitative polymerase chain reaction studies of volunteers challenged with malaria sporozoites. J Infect Dis 2005, 191:619-626.

8. Cheng Q, Lawrence G, Reed C, Stowers A, Ranford-Cartwright L, Creasey A Carter R, Saul A: Measurement of Plasmodium falciparum growth rate in vivo: a test of malaria vaccines. Am J Trop Med Hyg 1997, 57:495-500.

9. Hermsen CC, De Vlas SJ, Van Gemert GJA, Telgt DS, Verhage DF, Sauerwein RW: Testing vaccines in human experimental malaria; statistical analysis of parasitemia measured by a quantitative real-time polymerase chain reaction. Am J Trop Med Hyg 2004, 71:196-201.

10. Roestenberg M, O'Hara GA, Duncan CJ, Epstein JE, Edwards NJ, Scholzen A, van der Ven AJ, Hermsen CC, Hill AV, Sauerwein RW: Comparison of clinical and parasitological data from controlled human malaria infection trials. PLoS One 2012, 7:e38434

11. Powell RD, McNamara JV: Infection with chloroquine-resistant Plasmodium falciparum in man: prepatent periods, incubation periods, and relationships between parasitemia and the onset of fever in nonimmune persons. Ann NY Acad Sci 1970, 174:1027-1041.

12. Church LW, Le TP, Bryan JP, Gordon DM, Edelman R, Fries L, Davis JR, Herrington DA, Clyde DF, Shmuklarsky MJ, Schneider I, McGovern TW, Chulay JD, Ballou WR, Hoffman SL: Clinical manifestations of Plasmodium falciparum malaria experimentally induced by mosquito challenge. J Infect Dis 1997, 1997(175):915-920 
13. Epstein JE, Rao S, Williams F, Freilich $D$, Luke $T$, Sedegah $M$, de la Vega $P$, Sacci J, Richie TL, Hoffman SL: Safety and clinical outcome of experimental challenge of human volunteers with Plasmodium falciparum-infected mosquitoes: an update. J Infect Dis 2007, 196:145-154.

14. Roestenberg M, de Vlas SJ, Nieman AE, Sauerwein RW, Hermsen CC: Efficacy of preerythrocytic and blood-stage malaria vaccines can be assessed in small sporozoite challenge trials in human volunteers. $J$ Infect Dis 2013, 206:319-323.

15. Kamau E, Alemayehu S, Feghali KC, Saunders D, Ockenhouse CF: Multiplex qPCR for detection and absolute quantification of malaria. PLoS One 2013, 8:e71539.

16. Alemayehu S, Feghali KC, Cowden J, Komisar J, Ockenhouse CF, Kamau E: Comparative evaluation of published real-time PCR assays for the detection of malaria following MIQE guidelines. Malar J 2013, 12:277.

17. Kamau E, Tolbert LS, Kortepeter L, Pratt M, Nyakoe N, Muringo L, Ogutu B, Waitumbi JN, Ockenhouse CF: Development of a highly sensitive genus-specific quantitative reverse transcriptase real-time PCR assay for detection and quantification of Plasmodium by amplifying RNA and DNA of the 18S rRNA genes. J Clin Microbiol 2011, 49:2946-2953.

18. Douglas AD, Edwards NJ, Duncan CJ, Thompson FM, Sheehy SH, O'Hara GA, Anagnostou N, Walther M, Webster DP, Dunachie SJ, Porter DW, Andrews L, Gilbert SC, Draper SJ, Hill AV, Bejon P: Comparison of modeling methods to determine liver-to-blood inocula and parasite multiplication rates during controlled human malaria infection. J Infect Dis 2013, 208:340-345.

19. Schneider P, Wolters L, Schoone G, Schallig H, Sillekens P, Hermsen R, Sauerwein R: Real-time nucleic acid sequence-based amplification is more convenient than real-time PCR for quantification of Plasmodium falciparum. J Clin Microbiol 2005, 43:402-405.

20. Epstein JE: Taking a bite out of malaria: controlled human malaria infection by needle and syringe. Am J Trop Med Hyg 2013, 88:3-4.

doi:10.1186/1475-2875-13-288

Cite this article as: Kamau et al:: Measurement of parasitological data by quantitative real-time PCR from controlled human malaria infection trials at the Walter Reed Army Institute of Research. Malaria Journal 2014 13:288

\section{Submit your next manuscript to BioMed Central and take full advantage of:}

- Convenient online submission

- Thorough peer review

- No space constraints or color figure charges

- Immediate publication on acceptance

- Inclusion in PubMed, CAS, Scopus and Google Scholar

- Research which is freely available for redistribution 